\title{
Within-S ISI variations in GSR conditioning*
}

\author{
M. C. MORROW, H. H. RUETER \\ Georgia State University, Atlanta, Ga. 30303 \\ and \\ LORR L. W. KRAMER \\ Haverford College, Haverford, Pa. 19041
}

Twenty Ss received 15 trials a day for 2 days to each of two CSs. One CS (CS-S) was reinforced with a 1.0-sec ISI and the other (CS-L), with a 8.0-sec ISI. There were, in addition, five unreinforced presentations of each CS on each day. The major finding was that there was no overall difference in GSR magnitude to the two stimuli but that there was a significant ISI by Days interaction, i.e., GSR magnitude was greater to CS-L on Day 1 but smaller on Day 2 than it was to CS-S. The data supported the contention that the ISI variable behaves differently in the between- and within-S designs.

In a well-designed differential ISI conditioning study, Kimble, Leonard, \& Perlmuter (1968) found that, when two CSs were reinforced in the same $S$ but at different ISIs (one at 500 and the other at $1,200 \mathrm{msec}$ ), percentage of eyelid CRs was significantly greater to the long-interval CS than to the short- when the CRs in the last half of their respective ISIs were compared. The present experiment extended the differential ISI paradigm to the GSR and investigated the effects of ISI durations which have been shown, in this laboratory, to produce considerable differences in conditioning in the simple paradigm (Boring \& Morrow, 1968; Morrow \& Keough, 1968). In the latter respect, the study is in contrast to that by Kimble et al, in which ISI values were employed from which approximately equal performances have been customarily obtained (Kimble et al, 1968 , p. 654).

$$
\text { SUBJECTS }
$$

The Ss were 3 male and 17 female Georgia State University volunteer undergraduates. They were informed prior to participating that they would receive electric shock and were paid $\$ 3.00$ each. The instructions, which requested $S$ to remain motionless and pay attention to the stimuli, were read to him over an intercom system after he had been placed in the chamber referred to below.

\section{APPARATUS}

The equipment has been described elsewhere (Boring \& Morrow, 1968). Briefly, the CSs were two 2.54-cm-diam white lights, separated from each other by $2.54 \mathrm{~cm}$. They were presented on a black background at eye level, approximately $76 \mathrm{~cm}$ directly in front of $S$. The two CSs were easily differentiated spatially in the dimly lit sound-deadened experimental chamber. The UCS during conditioning was a $3.0-\mathrm{mA}$ $.1-\mathrm{sec}$ electric shock to the fingertips.

*Supported by PHS Grant MH 12065. closed the door. Approximately $3 \mathrm{~min}$ later, the instructions were read to the $\mathrm{S}$; then, after $2 \mathrm{~min}$, the experimental treatment began.

On Day 1, the $\mathrm{S}$ first received three UCS-alone trials with consecutive intensities of $1.5,2.0$, and $2.5 \mathrm{~mA}$. Next, he received four CS-alone trials, two with each CS in counterbalanced order. The duration of each CS during adaptation was $8.1 \mathrm{sec}$. Then $S$ received 15 reinforced trials to one $C S$ (CS-S) with a 1 -sec ISI (CS duration $=1.1 \mathrm{sec}$ ) and 15 reinforced trials to the other CS (CS-L) with an 8-sec ISI (CS duration $=8.1$ sec). Each CS (ISI) appeared once in each block of two trials, the CS on the first trial of a block determined by a coin flip. Additional CS-alone trials with CS-S were presented on chronological Trials $6,12,21,29$, and 40 and to CS-L on Trials 5, 14, 23, 30, and 39. Day 2 was like Day 1, except that no CS or UCS adaptation trials preceded the conditioning trials and only minimal additional instructions were provided. Test trials to CS-S occurred on Trials $6,15,21,31$, and 40 and to CS-L on Trials $8,13,22,30$, and 39 . Half of the Ss received the left light as CS-S and half the right. Intertrial intervals were 35,40 , and $45 \mathrm{sec}$, with a mean of $40 \mathrm{sec}$.

\section{RESULTS}

The GSR was recorded as a drop in resistance of at least $100 \mathrm{ohms}$ which began in the interval 1.5:5.5 sec after CS onset on the test trials. GSR magnitude to CS-S and CS-L across test trials on each day was examined using the entire sample, i.e., an ISI by Tests by Days analysis of variance. In inside. The CS projector was pointed out, and then $E$ left the chamber and then informed on Day 1 that he could speak to $E$ at any time over the intercom and that it was possible to intercom and that it was possible to

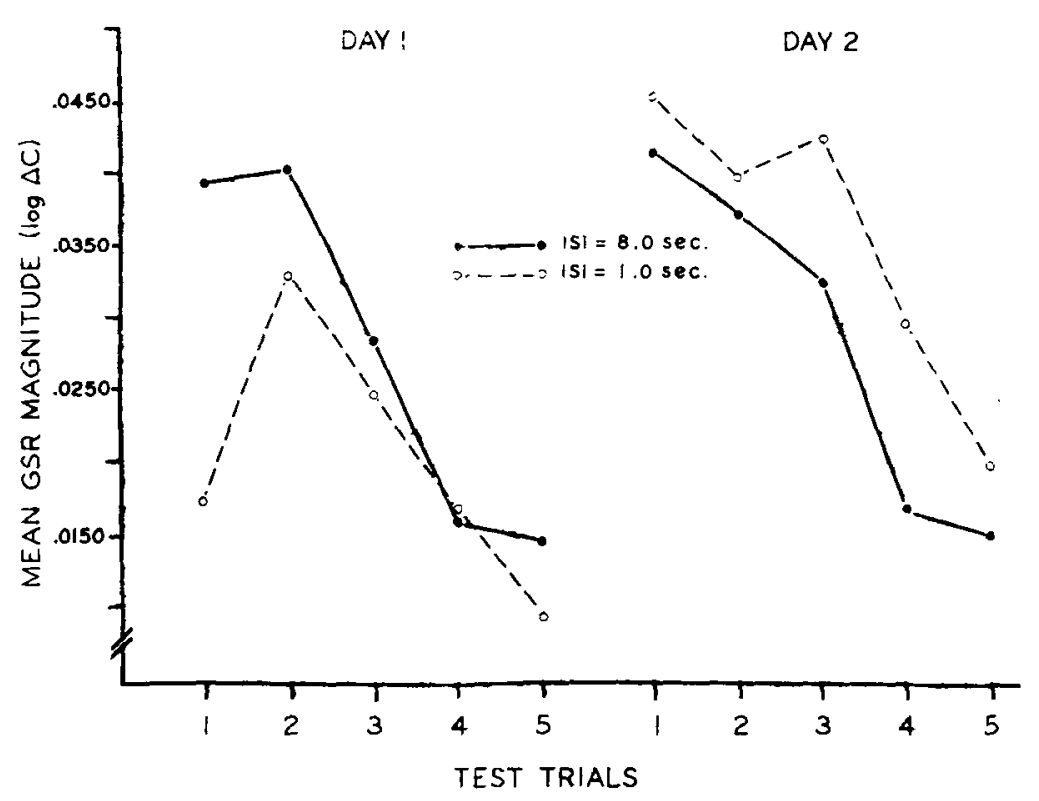

Fig. 1. Mean GSR magnitude $(\log \Delta \mathrm{C})$ on the test trials to each CS on Days 1 and 2 . 
Table 1


and Latency to CSS and CS-L on Days 1 and $2(N=16)$

\begin{tabular}{lcccc} 
& \multicolumn{2}{c}{ CS-S } & \multicolumn{2}{c}{ CS-L } \\
\cline { 2 - 5 } & Day 1 & Day 2 & Day 1 & Day 2 \\
\hline Magnitude & .0238 & .0440 & .0337 & .0357 \\
Amplitude & .0250 & .0446 & .0353 & .0364 \\
Percentage & $\mathbf{8 0 . 4 1}$ & $\mathbf{8 2 . 7 2}$ & $\mathbf{8 1 . 9 2}$ & $\mathbf{8 1 . 8 5}$ \\
Latency & $\mathbf{2 . 4 3}$ & $\mathbf{2 . 2 5}$ & $\mathbf{2 . 3 2}$ & $\mathbf{2 . 4 5}$ \\
\hline
\end{tabular}

addition, GSR magnitude, amplitude, percentage, and latency to each CS across days was evaluated, using the 16 Ss who gave at least one GSR to each C'S on each day, i.e., an ISI by Days analysis of variance. There were no differences in any measure between the Ss who received the left light as CS-S and those who received the right, so that the data of both subgroups were combined in all analyses. The resistance measures were transformed into units of log conductance change and the percentage measure into arcsin $\sqrt{\text { percentage }}$

Figure 1 shows the mean GSR magnitude on the test trials to each CS on both days. Mean magnitude was larger to CS-L than to CS-S on Day 1 , but the relationship was reversed on Day 2 so that there was little overall difference, combining days, between the CSs. Except for the discrepancy in magnitude to the CSs on the first test trial on Day 1, the performance curves for each CS were remarkably similar across test trials. The analysis showed that there was a significant decline in mean magnitude across tests $(F=13.33, \quad$ df $=4 / 361, \quad p<.01), \quad$ a significant overall increase in magnitude on the second day $(\mathrm{F}=10.83, \mathrm{df}=1 / 361, \mathrm{p}<.01)$, and $\mathrm{a}$ significant reversal in magnitude to the two stimuli from Day 1 to Day 2, i.e., a significant ISI by Days interaction $(\mathrm{F}=9.00, \mathrm{df}=1 / 361, \mathrm{p}<.01)$.

Mean GSR magnitude, amplitude, percentage, and latency to CS-S and CS-L on each day overall is shown in Table 1. The analyses of the magnitude, amplitude, and latency measures each produced a significant ISI by Days interaction effect (magnitude, $F=6.50 ;$ amplitude $F=14.00 ;$ latency, $F=5.43$; $\mathrm{df}=1 / 15, \mathrm{p}<.05$, for each test). The amplitude and magnitude interaction effects show that mean GSR was smaller to CS-S than to CS-L on Day 1, but larger on Day 2, and the latency interaction effect shows that mean latency was longer to CS-S than to CS-L on Day 1, but shorter on Day 2. Finally, mean GSR magnitude was greater on Day 2 overall than on Day $1(F=5.00, \mathrm{df}=1 / 15, \mathrm{p}<.05)$. There were no other significant effects in the data. The outcome of the magnitude, amplitude, and percentage analyses are interpreted to mean that the significant magnitude interaction and days effects were due to a modification in the size of the GSR rather than to a modification in the number of Ss responding.

Finally, the total number of second deflections to CS-S was 6 on Day 1 and 9 on Day 2. To CS-L, there were 39 on Day 1 and 46 on Day 2. These data are difficult to interpret, since CS-S was absent at the time of occurrence of the second deflection, while CS-L was present.

\section{DISCUSSION}

The data of this experiment are similar to those of Kimble et al (1968) in showing that the ISI variable behaves differently in the between-S and within-S designs. The usual between-S finding is that conditioning is greatest at. ISIs in the range of 400-500 msec (e.g., Kimble, 1947 Prokasy, Fawcett, \&. Hall, 1962) and declines rapidly at longer intervals (e.g., Fishbein \& LeBlanc, 1967; Morrow \& Keough, 1968). The present data, together with those of Kimble et al (1968) and Ominsky (1968), show that, in the within-s design, conditioning at longer ISIs is either equal to or greater than that at shorter ISIs and that the effect is the result of an attenuated response strength to the short-interval CS. Additionally, two other characteristics of the present data are particularly interesting: overall mean magnitude, on the one hand, and the slopes of the conditioning curves, on the other, more closely resemble data gathered in this laboratory in between-S designs from Ss receiving a long ISI (Morrow \& Keough, 1968) than from those receiving a short ISI (Boring \& Morrow, 1968). The ISI by Days interaction effects with the magnitude and amplitude measures suggest, however, that, were conditioning continued for a greater number of trials, the results would more closely approximate the typical findings of the between-S design.

The unusual findings of the within-s ISI paradigm do not appear to be due to some factor inherent in reinforcing both CSs. Support for this conclusion comes from several classical eyelid and GSR conditioning studies, in which both CSs were paired with UCSs (e.g.,
Grice \& Hunter, 1964; ̈̈ickens \& Harding, 1965). In these experiments, either CS or UCS intensity was the independent variable, and in each of the studies, the effects of the variable were the same, albeit, in one case greatly exaggerated, as they were when investigated with the simple conditioning paradigm. Thus, a major question arises: Why do intensity variables (CS, UCS intensity) behave in similar ways in both the between-S and within-S paradigms, but temporal variables (in this case, the ISI) do not?

Kimble et al (1968) presented a cognitive-perceptual account of their data which assumed that $\mathbf{S}$ must discriminate immediately after the presentation of a CS and that responses were inhibited until that presumably, immanent event was completed. It was not clear exactly which empirical events were being discriminated; at one time in the account it was the stimuli, i.e., one would guess, red vs green light; at another, it was the intervals, i.e., short vs long ISI (see p. 658). There are, of course, other possibilities. In any event, the present study was not designed to test their formulation. It is worth noting, however, that the Kimble et al theory does not handle the experiments of Grice \& Hunter (1964) and of Wickens \& Harding (1965). In those experiments, the $\mathbf{S}$ was presented with two reinforced stimuli and, presumably, thus was obliged to "discriminate" between them. Yet there was not an overall reduction in response strength during the early scoring interval (see different groups in Grice \& Hunter, 1964, for the necessary comparisons and compare Wickens \& Harding, 1965, to Wickens, Allen, \& Hill, 1963). In addition, if the time taken by $S$ to complete the discrimination were less than 1,000 msec-Kimble et al believe that it required more than $517 \mathrm{msec}$, and their data show that it surely required less than $1,217 \mathrm{msec}$-then there would have been time in the present experiment for CS-S to yet elicit a large response, since its duration was 1,100 msec.

Such an argument is, of course, in aid of proposing that the inhibitory discrimination hypothesis is perhaps not the most plausible account of the within-S ISI findings but, instead, that the findings may be more intimately related to the special characteristics of temporal variables in contrast to intensity variables. At any rate, a more direct evaluation of the Kimble et al hypothesis would come from a differential ISI design in which the $S$ was not required to discriminate. Data from an experiment in which $S$ is instructed before each trial regarding the CS and ISI to follow are currently being collected. 
REFERENCES

BORING, F. W., \& MORROW, M. C. Effects of UCS intensity upon conditioning and extinction of the GSR. Journal of Experimental Psychology, 1968, 77. 566-571.

FISHBEIN, H. D., \& LeBLANC, M. Human eyelid conditioning as a function of ISI. Joumal of Experimental Psychology $1967,75,130-133$.

GRICE, G. R., \& HUNTER, J. J. Stimulus intensity effects depend upon the type of experimental design. Psychological Review, 1964, 71, 247-256.

KIMBLE, G. A. Conditioning as a function of the time between conditioned and unconditioned stimuli. Journal of Experimental Psychology, 1947, 37, 1-15.

KIMBLE, G. A., LEONARD, T. B., III, \& PERLMUTER, L. C. Effects of interstimulus interval and discrimination learning in eyelid conditioning using between- and within-S designs. Journal of Experimental Psychology, 1968, 77, 65 2-660.
MORROW, M. C.. \& KEOUGH, T. E., III. GSR conditioning with long interstimulus intervals. Journal of Experimental Psychology, 1968, 77, 460-467.

OMINSKY, M. Differential instructions and interstimulus interval in eyelid conditioning. Psychonomic science, 1968. 12, 51-52.

PROKASY, W. F., FAWCETT, J. T., \& HALL, J, F. Recruitment, latency, magnitude, and amplitude of the GSR as a function of interstimulus interval. Journal of Experimental Psychology, $1962,64,513-518$.

WICKENS, D. D., ALLEN, C. K., \& HILL, F. A. Effect of instructions and UCS strength on extinction of the conditioned GSR. Journal of Experimental Psychology, 1963, 66, 235-240.

WICKENS, D. D., \& HARDING, G. B. Effect of UCS strength on GSR conditioning: A within-subject design. Journal of Experimental Psychology, $1965,70,151-153$.

\section{Interpersonal attraction as a function of attitude similarity dissimilarity and attitude extremity}

\author{
LEE A. JACKSON and GUILLERMO F. MASCARO \\ University of Florida, Gainesville, Fla. 32601
}

This experiment tested the effects of attitude extremity on the functional relationship between level of attitude similarity and interpersonal attraction. It was predicted that persons exhibiting greater attitude extremity would be more attracted to a "bogus" stranger who expressed similar attitudes and less attracted to a stranger expressing dissimilar attitudes than Ss holding more neutral attitudes. The results showed a significant main effect of attitude similarity on attraction, but the hypothesized interaction with attitude extremity was not found to be significant. The data were discussed in the context of other findings in the area.

Recent field and laboratory research across widely different experimental conditions has confirmed the hypothesis that interpersonal attraction is functionally related to attitude similarity (Berscheid \& Walster, 1969; Byrne, 1969; Newcomb, 1961). However, with the exception of a few studies (e.g., Byrne, 1965), there has been little focus on individual differences among $\mathrm{Ss}$ and their interactions with similarity in determining attraction. One of such parameters that needs to be investigated is the extremity of position of the experimental $S$ on the attitude items in which a stranger expresses attitude similarity or dissimilarity. addition, most authors consider those magnitudinal properties and their relationships to behavior to be among the most relevant functional characteristics of attitudes.

Interestingly enough, such a preeminent concept in the attitude literature as "topic importance" "has not been consistently found to affect the similarity-attraction relationship. In several incisive experiments, Byrne and his associates have demonstrated that the importance of the issue on which agreement occurred affected attraction (i.e., interacted with attitude similarity) only when the same person agreed/disagreed on items of different importance (Byrne, London, \& Griffitt, 1968; Clore \& Baldridge, 1968) but not when the importance of the items differed for different target persons and the items on which a given person agreed/disagreed had a uniform level of importance (Byrne \& Nelson, 1964,1965 ). Thus, apparently, the effects of topic importance, at least as a stimulus-manipulated variable, are limited only to certain paradigms of agreement-disagreement (e.g., the so-called intrastranger design).

Recently, Gormley \& Clore (1969) investigated the effects on attraction of agreement-disagreement in different items on which the Ss exhibited extreme or neutral responses. The types of items (i.e., extreme or neutral) on which a bogus stranger agreed/disagreed with the Ss did not affect attraction scores. However, this study did not address itself to determining the effects of attitude extremity as an organismic or individual-differences variable (i.e., the S's attitude extremity on a given issue).

The purpose of the present experiment is to test the effects of attitude extremity, as an organismic variable, on the similarity-attraction relationship. Based on the theoretically and empirically established relationship between extremity and other magnitudinal properties of attitudes (i.e., intensity, importance, degree of ego involvement, etc.), it can be predicted that the Ss' attitude extremity will affect the similarity-attraction function. Specifically, it is hypothesized that persons holding more extreme attitudes will develop greater attraction to those persons who express similar attitudes and lower attraction to those with discrepant attitudes than will persons who hold more neutral positions.

Since the "importance" variable has been found to be highly susceptible to experimental design variations (Byrne, 1969), testing the effects of extremity under the methodological conditions of this experiment (i.e., measuring 